\title{
Anaesthetic management of placenta accreta in hybrid operation theatre - A case report
}

\author{
Kritika Sharma ${ }^{1}$, Karnik Mamtora ${ }^{1}$, Sandip Katkade ${ }^{1}$, Tapas Mandal ${ }^{1}$, Hemant Mehta
}

\section{Abstract}

Introduction: Placenta accreta is a type of abnormal placentation where the placenta is adherent to the implantation site with an absent decidua and may produce life threatening challenges including major obstetric haemorrhage, need for peripartum hysterectomy and maternal and foetal morbidity and mortality. Although patients with placenta accreta are at high risk of massive haemorrhage, by using multi-disciplinary team approach and careful planning we can manage a patient with placenta accreta under neuraxial anaesthesia in hybrid operation theatre. We report a case of successful obstetric and anaesthetic management of a patient with diagnosed placenta accreta.

Keywords: placenta accreta, placenta increta, obstetric haemorrhage, hybrid theatre, multidisciplinary approach, subarachnoid block

\section{Introduction}

The placenta adheres to the decidua basalis layer, which allows for smooth separation from the uterine wall after delivery [1]. The term 'placenta accreta' is used when the placenta is adherent to the myometrium with absent decidual line of separation which can cause peripartum haemorrhage. Abnormal placentation is the most common indication for peripartum hysterectomy [2]. When not diagnosed early, it can lead to severe maternal morbidity.

Its incidence is approximately $0.04 \%$ and is steadily increasing as a recent study noted a rate of $0.12 \%$ [3]. It is severely affected by presence of placenta previa and the number of prior hysterotomies.

Perioperative management of placenta accreta and associated obstetric haemorrhage requires a multidisciplinary approach. We present a case of a patient with placenta accreta for elective caesarean section and the challenges faced by the anaesthesiologist during its management.

\section{Case Report}

A 29 year old female, G4P2L2A1, with placenta accreta presented for an elective caesarean section (CS) at 35 weeks of gestation. Her past obstetric history included two previous CS under subarachnoid block (SAB) and one abortion. Antenatal ultrasonography (USG) and Magnetic Resonance Imaging (MRI) was suggestive of grade four placenta previa with increta adherent to bladder in lower uterine segment. The patient had no other comorbidities. There was no history of antepartum haemorrhage. Foetal movements were satisfactory and estimated foetal weight was $2.13 \mathrm{~kg}$. Two doses of steroid were administered twelve hours apart for foetal lung maturity. She received intravenous (IV) iron therapy to improve the haemoglobin one week prior to the surgery.

Detailed preoperative evaluation was performed in advance, enabling a multidisciplinary team meeting. Thereby, a perioperative plan was formulated in close communication with the obstetric team, interventional radiologist, neonatologist, anaesthesiologist, intensivist, haematologist
${ }^{1}$ Department of Anaesthesia, Sir H N Reliance Foundation

Hospital, Mumbai, Maharashtra, India.

${ }^{2}$ Department of Anaesthesia, Apollo Hospitals, Ahmedabad, Gujarat, India.

Address of Correspondence

Dr. Kritika Sharma

Department of Anaesthesia, Sir H N Reliance Foundation

Hospital, Mumbai, Maharashtra, India.

E-mail: kriti2289@gmail.com

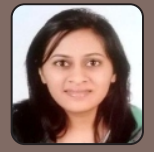

Dr. Kritika Sharma Dr. Karnik Mamtora

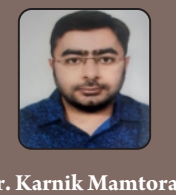

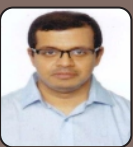

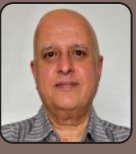

Dr. Hemant Mehta

Submitted: 2 September 2020; Reviewed: 21 November 2020; Accepted: 3 April 2021; Published: 10 May 2021

DOI: 10.13107/jaccr.2021.v07i02.177

This is an Open Access article distributed under the terms of the Creative Commons Attribution Non-Commercial-Share Alike 4.0 License (http://creativecommons.org/licenses/by-nc-sa/4.0) which allows others to remix, tweak, and build upon the work non-commercially as long as appropriate credit is given and the new creation are licensed under the identical terms. 


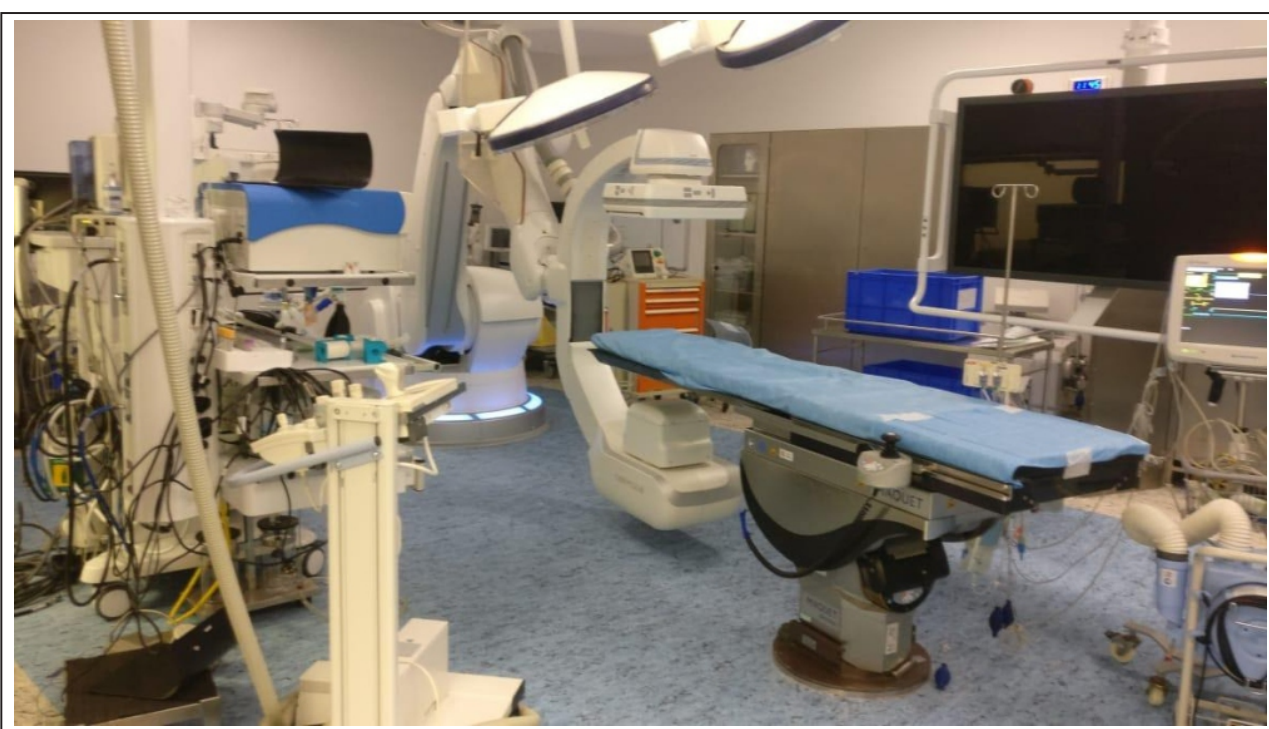

Figure 1: Hybrid operation theatre with facilities for both surgery and radiological intervention

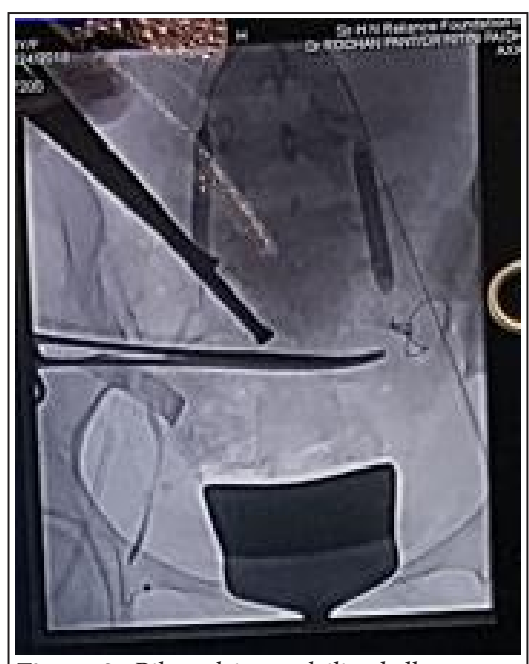

Figure 2: Bilateral internal iliac balloon were placed via femoral artery which were inflated immediately after delivery to reduce bleeding and blood bank. The anticipated duration of surgery of entire procedure as discussed in a multidisciplinary team meeting before the surgery was around $90-120$ minutes. Elective CS with internal iliac artery (IIA) balloon catheterisation and embolization with sos ligation with sos hysterectomy and oopherectomy was planned in the hybrid theatre under $\mathrm{SAB}$ with backup for general anaesthesia (GA). A written informed high risk consent was obtained. Adequate blood products were reserved, and the blood bank was informed about the possibility of massive blood transfusion and two packed cells were kept ready in the theatre. The hybrid theatre was prepared with required anaesthesia equipment, operating instruments and equipment for neonatal resuscitation.

On the day of surgery, in the hybrid theatre, two wide bore 18-gauge cannula were secured. Apart from the minimum mandatory ASA monitors, temperature, urine output, invasive blood pressure, central venous pressure and blood loss were monitored during surgery. Forced air warmer was applied and warm IV fluids were used. Under local anaesthesia, left radial 20 gauge arterial line for invasive blood pressure monitoring and triple lumen central venous line in right internal jugular vein was secured. SAB was given under strict asceptic precautions in sitting position using Whitacre needle at L3-4 level and $2 \mathrm{ml}$ of $0.5 \%$ hyperbaric Bupivacaine with $20 \mathrm{mcg}$ Fentanyl was injected in intrathecal space. We did not give a higher dose to avoid any hemodynamic instability in case of a massive haemorrhage. Also, a backup for general anaesthesia was kept ready in case of prolonged duration of surgery or any hemodynamic instability. The patient was made supine immediately after giving $S A B$ and IIA balloon placement was done via right femoral artery by interventional radiologist.
The surgeons took a Pfannensteil incision and excised the adhesions between the uterus and anterior abdominal wall. Bladder was adherent to the lower uterine segment which was pushed down after careful blunt dissection.

Placental invasion in the anterior wall of uterus was observed. Uterine incision was made in the lower uterine segment and a healthy female baby was delivered after 18 minutes of SAB. IIA balloon was inflated soon after delivery to reduce the bleeding and embolization was done as shown in Figure 2. The placenta was adherent and removed in pieces. There was no bladder invasion. The part of the lower uterine wall with placental invasion was excised and uterus was closed. To control the bleeding further, B Lynch sutures were applied. $200 \mathrm{mcg}$ intramuscular Methylergometrine and $800 \mathrm{mcg}$ sublingual Misoprostol were administered. Abdominal drain was inserted before closure.

Intraoperatively, $2000 \mathrm{ml}$ of crystalloids, $500 \mathrm{ml}$ of colloids and 1 unit packed red cells were given. The estimated blood loss was $1500 \mathrm{ml}$. Urine output was adequate. Patient was shifted to ICU for observation. Patient was kept in ICU for one day and discharged from the hospital on sixth postoperative day.

\section{Discussion}

Abnormal placentation including placenta previa and placenta accreta is one of the leading causes of peripartum haemorrhage and is the most common indication of peripartum hysterectomy [2]. Placenta previa includes a lowlying placenta, either covering or encroaching the cervical os with its incidence being roughly $0.5 \%$ of all pregnancies. It is more common in multiparous women, advanced maternal age, assisted pregnancy, prior placenta previa, and uterine scarring from prior surgery or infection. The term placenta 
accreta includes three subtypes-accreta vera, increta, and percreta. Placenta accreta vera is an abnormal adherence to the myometrium with an abnormal decidual line of separation. Placenta increta is abnormal implantation and growth of placenta into the myometrium and placenta percreta is placental growth through the uterine wall and implantation onto organs surrounding the uterus like bladder, bowel, ovaries. The incidence of placenta accreta is severely affected by the presence of placenta previa and previous hysterotomies. Our patient was diagnosed with placenta previa with increta and had undergone two previous caesarean sections.

Although the overall incidence of placenta accreta/increta is veryless, but in patients with known placenta previa, the rates of accreta are recorded to be $3 \%, 11 \%, 40 \%$ and more than $60 \%$ with zero, one, two and three or more prior uterine incisions [4]. USG and/or MRI are used to diagnose placenta accreta. In our patient, USG revealed grade four placenta previa with increta adherent to bladder in lower uterine segment which was confirmed by MRI.

Multidisciplinary team with obstetricians, anaesthetists, radiologists, neonatologists, haematologists, urologists, and gynaecology surgeon is needed in these patients. Given the possibility of massive blood loss, meticulous planning and management with an elective caesarean delivery and if required peripartum hysterectomy is needed. Sometimes placenta may be left in situ. Oxygen carrying capacity of blood can be optimized with oral iron therapy as in our patient. A mother with abnormal placenta should be managed in a tertiary care hospital with all facilities to manage a massive obstetric haemorrhage.

Most anaesthetists prefer GA in these cases as it allows better control of ventilation and better hemodynamic stability during a massive haemorrhage but neuraxial anaesthesia with standby GA can also be given safely, provided adequate precautions are taken to avoid hypoxia and hypotension and control major bleeding. $\mathrm{SAB}$ provides anaesthesia for the placement of the femoral arterial sheath as well as postoperative analgesia along with the advantages of decreased risk of aspiration, minimizing blood loss, allowing the bonding of mother and baby, and reducing the foetal exposure to anaesthetic drugs. Simultaneously, to avoid any haemodynamic instability and massive haemorrhage IIA balloon catheter was placed by the interventional radiologist after giving spinal which was inflated immediately after delivery followed by embolization.

Many cases have been reported where the patient is transported from the operation theatre to the interventional radiology suite immediately after delivery for embolization. Tasneem et al reported a case of caesarean section, in a patient with placenta percreta and described the challenges faced by anaesthesiologist during its management including preparation for massive blood loss, providing anaesthesia in a remote location, and transport of an anaesthetised patient back and forth between interventional radiology suite and the operation theatre [5].

Such back and forth transportation can be difficult in a bleeding patient and also carries the risk of displacement and accidental removal of sheath during transportation.

To avoid such mishaps, it is best to carry out these surgeries in the hybrid operation theatre if available, which provides optimal conditions for the surgery and radiological intervention.

A case series of thirteen patients was reported by Heidemann et al of patients with suspected placenta accrete or percreta who underwent caesarean sections with peri-operative bilateral internal iliac artery catheterization with or without balloon occlusion or embolisation and concluded that it improves the operative field and reduces blood loss and transfusion requirements [6].

However, all necessary arrangements must be made including availability of all operating instruments, neonatal resuscitation, necessary drugs and equipment for general anaesthesia and adequate blood supply. Communication with the blood bank and activation of massive transfusion protocol ensures prompt availability of blood and other products until the bleeding can be stopped surgically. This can be lifesaving in a situation of massive haemorrhage. In our case, we were ready with two packed cells but as the bleeding was controlled well by surgical means, only one packed cell was transfused.

Although there has been a concern about using Intraoperative Cell Salvage (ICS) in pregnant women because of the theoretical risk of amniotic fluid embolus (AFE), there have been no reported cases of AFE attributed to ICS use in obstetrics $[7,8,9]$. The use of ICS has been advocated by the American College of Obstetrics and Gynaecology for mothers in whom massive bleeding is anticipated, such as those with placenta accreta.

Recombinant factor VIIa has also been used off-label to treat major postpartum haemorrhages with positive results but it should be cautiously used because of risk of thrombosis [10]. Adequate measures must be taken to avoid hypothermia which can worsen coagulopathy, acidosis and bleeding. Thus in our case, by using multi-disciplinary team approach and careful planning we were able to manage a patient with placenta accreta under neuraxial anaesthesia with a good outcome.

\section{Conclusion}

Patients with placenta accreta are at high risk of massive haemorrhage. Its management requires early detection, 
planning and team approach. This can be achieved by preoperative placement of internal iliac artery balloon catheter by the interventional radiologist which can be inflated immediately after delivery and embolization, careful dosing of local anaesthetic for spinal anaesthesia, careful positioning, strict haemodynamics monitoring and surgical control of bleeding which may need a hysterectomy if not controlled.

Declaration of patient consent: The authors certify that they have obtained all appropriate patient consent forms. In the form, the patient has given his consent for his images and other clinical information to be reported in the Journal. The patient understands that his name and initials will not be published, and due efforts will be made to conceal his identity, but anonymity cannot be guaranteed.

\section{Conflict of interest: Nil Source of support: None}

\section{References}

1. Placenta accreta and anesthesia: A multidisciplinary approach RS Khokhar,J Baaj, MU Khan, FA Dammas, and N Rashid Author information Copyright and License information Disclaimer

2. Wright JD, Devine P, Shah M, Gaddipati S, Lewin SN, Simpson LL, et al. Morbidity and mortality of peripartum hysterectomy. Obstet Gynecol. 2010;115:1187-93. [PubMed] [Google Scholar]

3. Miller's Anesthesia, 8e, Ch 77. Anesthesia for Obstetrics 2351

4. Silver RM, Landon MB, Rouse DJ, Leveno KJ, Spong CY, Thom EA, et al. National Institute of Child Health and Human Development Maternal-Fetal Medicine Units Network. Maternal morbidity associated with multiple repeat cesarean deliveries. Obstet Gynecol. 2006;107:1226-1232. [PubMed] [Google Scholar]

5. Dhansura, Tasneem et al. "Anaesthesiologist's role in the multidisciplinary approach to placenta percreta." Indian journal of anaesthesia vol. 59,8 (2015): 513-5. doi:10.4103/0019-5049.163002

6. Mok M, Heidemann B, Dundas K, Gillespie I, Clark V. Interventional radiology in women with suspected placenta accreta undergoing caesarean section. Int $\mathrm{J}$ Obstet Anesth. 2008 Jul;17(3):255-61. doi: 10.1016/j.ijoa.2007.11.010. Epub 2008 Jun 2. PMID: 18513942.
7. Khan, K, Moore P, Wilson M, et al. Cell Salvage and donor blood transfusion during caesarean section: A pragmatic, multicenter randomized controlled trail ( S A L V O ). P L o S M e d 14 ( $\left.\begin{array}{ll}1 & 2\end{array}\right)$ : e 1002471 . https://doi.org/10.1371/journal.pmed.1002471

8. Corfe J. Joint guideline on the management of intraoperative cell salvage in obstetrics. 2017. Norfolk and Norwich University Hospitals, Norwich, England.

9. Goucher H, Wong C, Patel S et al. Cell salvage in obstetrics. Anesth Analg. 2015;121:465-468.

10. Franchini M, Franchi M, Bergamini V, Salvagno GL, Montagnana M, Lippi G, et al. A critical review on the use of recombinant factor VIIa in life-threatening obstetric postpartum hemorrhage. Semin Thromb Hemost 2008;34:104-12.

\section{How to Cite this Article}

Sharma K, Mamtora K, Katkade S, Mandal T, Mehta H | Anaesthetic management of placenta accreta in hybrid operation theatre - A case report | Journal of Anaesthesia and Critical Care Case Reports | May-August 2021; 7(2): 21-24. 